\title{
Worldsheet (anti)instanton bound states in type II on $T^{2}$
}

\author{
Anirban Basu \\ Harish-Chandra Research Institute, HBNI, \\ Chhatnag Road, Jhusi, Prayagraj 211019, India \\ E-mail: anirbanbasu@hri.res.in
}

ABSTRACT: The $1 / 8$ BPS $D^{6} \mathcal{R}^{4}$ coupling in type II string theory compactified on $T^{2}$ receives contributions from worldsheet instantons and anti-instantons wrapping the $T^{2}$, up to genus three in string perturbation theory. These involve contributions separately from bound states of instantons and anti-instantons, which are qualitatively similar to such contributions to the $1 / 2$ and $1 / 4$ BPS couplings. At genus two, the $D^{6} \mathcal{R}^{4}$ coupling also receives contributions from instanton/anti-instanton bound states unlike the $1 / 2$ and $1 / 4$ BPS couplings, which is a consequence of a T-duality invariant eigenvalue equation a term in the coupling satisfies. We solve this eigenvalue equation to obtain the complete structure of the worldsheet (anti)instanton contributions. In the type IIB theory, strong weak coupling duality leads to certain contributions involving bound states of D string (anti)instantons wrapping the $T^{2}$.

KEYWORDs: Extended Supersymmetry, Supersymmetry and Duality

ArXiv EPrint: 2003.03301 


\section{Contents}

1 Introduction $\quad 1$

2 The analysis of the eigenvalue equation for $F(T, \bar{T}) \quad 3$

2.1 The mode carrying no NS charge 4

2.2 The modes carrying NS charge 5

2.2.1 The solution for $I_{n}\left(T_{2}\right) \quad 6$

$\begin{array}{ll}2.2 .2 \text { The solution for } I_{n_{1}, n_{2}}\left(T_{2}\right) & 7\end{array}$

3 S-duality and an elementary consequence for $D$ string instanton contributions

A The $\mathrm{SL}(2, \mathbb{Z})$ invariant non-holomorphic Eisenstein series 11

$\begin{array}{ll}\text { B The exponential integral function } & 11\end{array}$

\section{Introduction}

Consider type II string theory toroidally compactified on $T^{2}$. This maximally supersymmetric theory has a U-duality symmetry group $\mathrm{SL}(2, \mathbb{Z}) \times \mathrm{SL}(3, \mathbb{Z})$. In the type IIB theory, the non-perturbative ${ }^{1} \mathrm{SL}(2, \mathbb{Z})_{\tau}$ S-duality symmetry which is inherited from ten dimensions is contained in $\mathrm{SL}(3, \mathbb{Z})$ of the U-duality group. The perturbative T-duality symmetry group is

$$
\mathrm{SL}(2, \mathbb{Z})_{T} \times \mathrm{SL}(2, \mathbb{Z})_{U}
$$

where $T$ and $U$ are the complexified Kahler and complex structure moduli of the $T^{2}$ respectively. While $\operatorname{SL}(2, \mathbb{Z})_{U}$ directly arises as the $\operatorname{SL}(2, \mathbb{Z})$ factor in the U-duality group, the $\mathrm{SL}(2, \mathbb{Z})_{T}$ is contained in $\mathrm{SL}(3, \mathbb{Z})$. The moduli dependent coefficients of various amplitudes in this theory when expanded around weak string coupling exhibit a rich perturbative as well as non-perturbative structure.

In the string frame, the perturbative part of the amplitude takes the form

$$
\sum_{g}\left(e^{-2 \phi} V\right)^{1-g} f_{g}(T, \bar{T} ; U, \bar{U})
$$

where $\phi$ is the dilaton, and $V$ is the volume of $T^{2}$ in the string frame metric. The Kahler modulus is given by

$$
T=T_{1}+i T_{2}=B_{N}+i V
$$

\footnotetext{
${ }^{1}$ Here perturbative and non-perturbative are with respect to the string coupling.
} 
where $B_{N}$ is the scalar from the NS-NS sector. In (1.2), the term involving $f_{g}$ is the genus $g$ amplitude, which involves the T-duality invariant string coupling $e^{-2 \phi} V$ as the overall factor. Equality of the IIA and IIB theories on compactifying on $T^{2}$ yields that $f_{g}(T, \bar{T} ; U, \bar{U})=f_{g}(U, \bar{U} ; T, \bar{T})$. Note that the perturbative contribution (1.2) does not involve states from the Ramond sector.

The non-perturbative contributions arise from D-instantons as well as from $(p, q)$ string instantons $^{2}$ wrapping $T^{2}$ where $q \neq 0$, and are exponentially suppressed for large $\tau_{2}$. For the $n$ D-instanton contribution, the exponentially suppressed factor is of the form

$$
e^{2 \pi i\left(n \tau_{1}+i|n| \tau_{2}\right)}
$$

while for the $(p, q)$ string instanton contribution, it is of the form $[1,2]$

$$
e^{2 \pi i T_{p, q}}
$$

where $T_{p, q}=\left(q B_{R}+p B_{N}\right)+i|p-q \tau| V$, where $B_{R}$ is the scalar from the R-R sector. While the instantons carry positive NS (or R) charge, the anti-instantons carry negative charge.

Let us consider the perturbative contributions given by (1.2). Though they are perturbative in the string coupling, they can receive contributions which are non-perturbative in $\alpha^{\prime}$, the inverse string tension. These contributions arise from worldsheet instantons and anti-instantons ${ }^{3}$ wrapping $T^{2}$. While it is difficult to calculate these contributions for generic interactions, the BPS interactions are amenable to a detailed analysis.

First let us consider the $1 / 2$ BPS $\mathcal{R}^{4}$ interaction, where only the terms involving $g=0$ and 1 are non-vanishing in (1.2). The worldsheet (anti)instanton contributions are given by $[2-4]$

$$
f_{1}=2 \pi \sum_{n=1}^{\infty} \frac{\sigma_{1}(n)}{n}\left(e^{2 \pi i n T}+e^{-2 \pi i n \bar{T}}\right),
$$

where we have ignored all other contributions. ${ }^{4}$

For the $1 / 4$ BPS $D^{4} \mathcal{R}^{4}$ interaction, where only terms involving $g=0,1$ and 2 are non-vanishing in (1.2), the worldsheet (anti)instanton contributions are given by $[4,5]$

$$
\begin{aligned}
& f_{1}=\frac{4}{\pi} E_{2}(U, \bar{U}) \sum_{n=1}^{\infty} \frac{\sigma_{3}(n)}{n^{2}}\left(1+\frac{1}{2 \pi n T_{2}}\right)\left(e^{2 \pi i n T}+e^{-2 \pi i n \bar{T}}\right), \\
& f_{2}=\frac{4 \pi^{2}}{3} \sum_{n=1}^{\infty} \frac{\sigma_{3}(n)}{n^{2}}\left(1+\frac{1}{2 \pi n T_{2}}\right)\left(e^{2 \pi i n T}+e^{-2 \pi i n \bar{T}}\right)
\end{aligned}
$$

where the Eisenstein series $E_{2}$ is defined by (A.1) and we have ignored all other contributions. ${ }^{5}$

Thus (1.6) and (1.7) both involve an infinite sum of worldsheet (anti)instanton contributions. In fact, each term in the sum results from either instantons or from anti-instantons.

\footnotetext{
${ }^{2}$ We follow the convention of denoting the fundamental string as the $(1,0)$ state.

${ }^{3}$ In fact, (1.5) also contains such contributions for $p \neq 0$. However, for the sake of simplicity we restrict ourselves to contributions involving no Ramond sector states.

${ }^{4}$ Our normalization is such that $f_{0}=\zeta(3)$.

${ }^{5}$ Our normalization is such that $f_{0}=\zeta(5)$.
} 
This feature changes qualitatively when we consider the $1 / 8 \mathrm{BPS} D^{6} \mathcal{R}^{4}$ interaction which preserves 4 supercharges. This interaction receives contributions from $g=0,1,2$ and 3 in (1.2). Again, keeping only terms involving the worldsheet (anti)instanton contributions, we have that $[4,6]^{6}$

$$
\begin{aligned}
f_{1}= & \frac{10}{\pi^{2}} E_{3}(U, \bar{U}) \sum_{n=1}^{\infty} \frac{\sigma_{5}(n)}{n^{3}}\left(1+\frac{3}{2 \pi n T_{2}}+\frac{3}{4 \pi^{2} n^{2} T_{2}^{2}}\right)\left(e^{2 \pi i n T}+e^{-2 \pi i n \bar{T}}\right) \\
& +2 \pi \zeta(3) \sum_{n=1}^{\infty} \frac{\sigma_{1}(n)}{n}\left(e^{2 \pi i n T}+e^{-2 \pi i n \bar{T}}\right) \\
f_{2}= & 2 \pi\left(E_{1}(U, \bar{U})+\frac{\pi}{6}\right) \sum_{n=1}^{\infty} \frac{\sigma_{1}(n)}{n}\left(e^{2 \pi i n T}+e^{-2 \pi i n \bar{T}}\right)+F(T, \bar{T}), \\
f_{3}= & \frac{\pi^{3}}{9} \sum_{n=1}^{\infty} \frac{\sigma_{5}(n)}{n^{3}}\left(1+\frac{3}{2 \pi n T_{2}}+\frac{3}{4 \pi^{2} n^{2} T_{2}^{2}}\right)\left(e^{2 \pi i n T}+e^{-2 \pi i n \bar{T}}\right) .
\end{aligned}
$$

In (1.8), $F(T, \bar{T})$ satisfies the eigenvalue equation

$$
(\Delta-12) F(T, \bar{T})=-6\left(E_{1}(T, \bar{T})\right)^{2}
$$

where

$$
\Delta=4 T_{2}^{2} \frac{\partial^{2}}{\partial T \partial \bar{T}}
$$

is the $\operatorname{SL}(2, \mathbb{Z})_{T}$ invariant Laplacian. The relevant Eisenstein series that appear in (1.8) are defined by (A.1) and (A.3). The 1/8 BPS couplings have also been analyzed from the worldsheet perspective in [7-11], and from the spacetime point of view in [12-23].

Now in (1.8) all the contributions apart from that involving $F(T, \bar{T})$ are given by an infinite sum of terms involving either worldsheet instantons or anti-instantons. However, while $F(T, \bar{T})$ yields qualitatively similar contributions separately from bound states of instantons or anti-instantons, it receives additional contributions involving bound states of instantons/anti-instantons because of the presence of the source term in the eigenvalue equation (1.9). In this paper, we analyze the content of (1.9) in detail to understand all these contributions at a quantitative level.

\section{The analysis of the eigenvalue equation for $F(T, \bar{T})$}

We now analyze the eigenvalue equation (1.9) in detail. To start with, we express $F(T, \bar{T})$ as

$$
F(T, \bar{T})=\sum_{n \in \mathbb{Z}} F_{n}\left(T_{2}\right) e^{2 \pi i n T_{1}}
$$

This involves an infinite sum over topologically distinct sectors carrying non-trivial NS charge (the $n=0$ sector carries no charge).

We shall solve (1.9) along with specific boundary conditions. For large $T_{2}$, we have that $F(T, \bar{T}) \sim T_{2}^{2}$ simply because this contribution arises at genus two and this is the

\footnotetext{
${ }^{6}$ Our normalization is such that $f_{0}=\zeta(3)^{2}$.
} 
large volume scaling. For small $T_{2}$, the large $T_{2}$ behavior along with $\operatorname{SL}(2, \mathbb{Z})_{T}$ invariance yields that $[24]$

$$
F_{n}\left(T_{2}\right) \sim T_{2}^{-1}
$$

for all $n$.

\subsection{The mode carrying no NS charge}

First let us consider the mode $F_{0}\left(T_{2}\right)$ in (2.1) which carries no NS charge. Using (1.9) and the large $T_{2}$ expansion of $E_{1}$ given in (A.3), we have that

$$
\left(T_{2}^{2} \frac{d^{2}}{d T_{2}^{2}}-12\right) F_{0}\left(T_{2}\right)=-6\left(\frac{\pi^{2}}{3} T_{2}-\pi \ln T_{2}\right)^{2}-48 \pi^{2} \sum_{n=1}^{\infty} \frac{\sigma_{1}(n)^{2}}{n^{2}} e^{-4 \pi n T_{2}} .
$$

While the solution of the homogeneous equation is given by ${ }^{7}$

$$
F_{0}^{H}\left(T_{2}\right)=a_{0} T_{2}^{-3},
$$

where $a_{0}$ is an arbitrary constant, the particular solution is given by [6]

$$
\begin{aligned}
F_{0}^{P}\left(T_{2}\right)= & \frac{\pi^{2}}{720}\left(65-20 \pi T_{2}+48 \pi^{2} T_{2}^{2}\right)+\pi^{2} \ln T_{2}\left(-\frac{\pi}{3} T_{2}+\frac{1}{2} \ln T_{2}-\frac{1}{12}\right) \\
& +\sum_{n=1}^{\infty} Q_{n}\left(T_{2}\right) e^{-4 \pi n T_{2}} .
\end{aligned}
$$

In (2.5), $Q_{n}\left(T_{2}\right)$ is given by

$$
Q_{n}\left(T_{2}\right)=-\frac{\sigma_{1}(n)^{2}}{224 n^{5} \pi T_{2}^{3}}\left[24(x+1)^{2}+x^{4}(2-x)+\left(x^{3}-3\right)^{2}+15+x^{7} e^{x} \operatorname{Ei}(-x)\right],
$$

where $x=4 \pi n T_{2}$ and $\operatorname{Ei}(-x)$ is the exponential integral function defined in (B.1). Using (B.2), we see that $Q_{n}\left(T_{2}\right)$ is an infinite series in powers of $T_{2}$ in the large $T_{2}$ expansion. Since this contribution is weighted by $e^{-4 \pi n T_{2}}$, it follows that it arises from the bound state of worldsheet instantons/anti-instantons carrying equal and opposite NS charge $n$.

We now fix $a_{0}$ in (2.4) using the boundary condition (2.2). Using (B.3) we see that the singular terms in the small $T_{2}$ limit in $F_{0}^{P}\left(T_{2}\right)$ are given by

$$
F_{0}^{P}\left(T_{2}\right)=-\frac{3}{14 \pi T_{2}^{3}} \sum_{n=1}^{\infty} \frac{\sigma_{1}(n)^{2}}{n^{5}}+\frac{\pi^{2}}{2} \ln T_{2}\left(\ln T_{2}-\frac{1}{6}\right)+O\left(T_{2}^{0}\right) .
$$

While the $O\left(T_{2}^{-3}\right)$ contribution comes from the worldsheet instanton/anti-instanton sector, the $O\left(T_{2}^{-2}\right)$ and $O\left(T_{2}^{-1}\right)$ terms that arise from this sector cancel on adding the various contributions. Now using the relation

$$
\sum_{n=1}^{\infty} \frac{\sigma_{p}(n) \sigma_{q}(n)}{n^{r}}=\frac{\zeta(r) \zeta(r-p) \zeta(r-q) \zeta(r-p-q)}{\zeta(2 r-p-q)}
$$

\footnotetext{
${ }^{7}$ We neglect the solution $T_{2}^{4}$ as it violates the large $T_{2}$ boundary condition.
} 
we see that (2.7) yields

$$
F_{0}^{P}\left(T_{2}\right)=-\frac{\zeta(3) \zeta(5)}{4 \pi T_{2}^{3}}+\frac{\pi^{2}}{2} \ln T_{2}\left(\ln T_{2}-\frac{1}{6}\right)+O\left(T_{2}^{0}\right) .
$$

Thus demanding the cancellation of the $O\left(T_{2}^{-3}\right)$ terms between $F_{0}^{H}\left(T_{2}\right)$ and $F_{0}^{P}\left(T_{2}\right)$ for small $T_{2}$, we get that

$$
a_{0}=\frac{\zeta(3) \zeta(5)}{4 \pi} .
$$

This precisely agrees with this result obtained using a different method in [6]. Hence for small $T_{2}$, the singularity in $F_{0}\left(T_{2}\right)$ is only logarithmic, and is weaker than the bound in (2.2).

Thus the complete solution is given by

$$
F_{0}\left(T_{2}\right)=F_{0}^{H}\left(T_{2}\right)+F_{0}^{P}\left(T_{2}\right)
$$

on using (2.4), (2.5) and (2.10).

When expanded for large $T_{2}$, we see that $F_{0}\left(T_{2}\right)$ has terms that are power behaved and logarithmic in $T_{2}$, as well as terms that are exponentially suppressed in $T_{2}$. In the large $T_{2}$ expansion of $Q_{n}\left(T_{2}\right)$ in (2.6), on using (B.2) we see that there are several cancellations at leading orders, which yield the leading contribution

$$
-\frac{3 \sigma_{1}(n)^{2}}{n^{4} T_{2}^{2}} e^{-4 \pi n T_{2}}
$$

to $F_{0}\left(T_{2}\right)$ from the instanton/anti-instanton sector with weight $e^{-4 \pi n T_{2}}$.

\subsection{The modes carrying NS charge}

We now consider the modes in (2.1) that carry non-vanishing NS charge. We express the mode $F_{n}\left(T_{2}\right)(n \neq 0)$ which carries $n$ units of NS charge as

$$
F_{n}\left(T_{2}\right)=I_{n}\left(T_{2}\right)+\sum_{n_{i} \neq 0, n_{1}+n_{2}=n} I_{n_{1}, n_{2}}\left(T_{2}\right),
$$

where $I_{n}\left(T_{2}\right)$ and $I_{n_{1}, n_{2}}\left(T_{2}\right)$ satisfy the differential equations

$$
\left(T_{2}^{2} \frac{d^{2}}{d T_{2}^{2}}-12-4 \pi^{2} n^{2} T_{2}^{2}\right) I_{n}\left(T_{2}\right)=-\frac{24 \pi^{2} \sigma_{1}(n)}{|n|}\left(\frac{\pi}{3} T_{2}-\ln T_{2}\right) e^{-2 \pi|n| T_{2}}
$$

and

$$
\left(T_{2}^{2} \frac{d^{2}}{d T_{2}^{2}}-12-4 \pi^{2}\left(n_{1}+n_{2}\right)^{2} T_{2}^{2}\right) I_{n_{1}, n_{2}}\left(T_{2}\right)=-\frac{24 \pi^{2} \sigma_{1}\left(n_{1}\right) \sigma_{1}\left(n_{2}\right)}{\left|n_{1} n_{2}\right|} e^{-2 \pi\left(\left|n_{1}\right|+\left|n_{2}\right|\right) T_{2}}
$$

respectively.

We now solve (2.14) and (2.15) with appropriate choice of boundary conditions. For large $T_{2}$, the solutions $I_{n}\left(T_{2}\right)$ and $I_{n_{1}, n_{2}}\left(T_{2}\right)$ must have a growth no faster than $T_{2}^{2}$ for the same reasons as before. ${ }^{8}$ For small $T_{2}$ each mode has singular behavior no worse than $T_{2}^{-1}$ in order to satisfy $(2.2)$.

\footnotetext{
${ }^{8}$ In fact, we shall see that the solutions are exponentially suppressed, hence exhibiting significantly milder behavior.
} 


\subsubsection{The solution for $I_{n}\left(T_{2}\right)$}

We express

$$
I_{n}\left(T_{2}\right)=I_{n}^{H}\left(T_{2}\right)+I_{n}^{P}\left(T_{2}\right),
$$

where $I_{n}^{H}\left(T_{2}\right)$ is a solution to the homogeneous equation (2.14), while $I_{n}^{P}\left(T_{2}\right)$ solves the particular equation (2.14).

Now $I_{n}^{H}\left(T_{2}\right)$ is given by [24]

$$
I_{n}^{H}\left(T_{2}\right)=b_{n} \sqrt{T_{2}} K_{7 / 2}\left(2 \pi|n| T_{2}\right)
$$

where $b_{n}$ is an arbitrary constant. We ignore the other solution $\sqrt{T_{2}} I_{7 / 2}\left(2 \pi|n| T_{2}\right)$ since it grows exponentially for large $T_{2}$, violating our boundary condition.

The particular solution $I_{n}^{P}\left(T_{2}\right)$ is given by

$$
\begin{aligned}
I_{n}^{P}\left(T_{2}\right)= & -\frac{\sigma_{1}(n) e^{-2 \pi|n| T_{2}}}{16 \pi n^{4} T_{2}^{3}}\left[-12\left(2 x^{2}+5 x+5\right) \ln (x / 2 \pi|n|)\right. \\
& -\frac{4}{|n|} P(x) \ln x+4\left(1+\frac{1}{|n|}\right) P(-x) e^{2 x} \operatorname{Ei}(-2 x) \\
& \left.-\left(26 x^{2}+95 x+215\right)-\frac{4}{|n|}\left(7 x^{2}+25 x+55\right)\right],
\end{aligned}
$$

where $x=2 \pi|n| T_{2}$, and $P(x)$ is a polynomial in $x$ defined by

$$
P(x)=x^{3}+6 x^{2}+15 x+15 .
$$

To determine $b_{n}$ using the boundary condition at small $T_{2}$ mentioned above, we expand both $I_{n}^{H}\left(T_{2}\right)$ in $(2.17)$ and $I_{n}^{P}\left(T_{2}\right)$ in (2.18) for small $T_{2}$. For the solution to the homogeneous equation, we have that

$$
I_{n}^{H}\left(T_{2}\right)=b_{n}\left[\frac{15}{16|n|^{7 / 2} \pi^{3} T_{2}^{3}}\left(1-\frac{2}{5} \pi^{2} n^{2} T_{2}^{2}\right)+O\left(T_{2}\right)\right] .
$$

For the particular solution we get

$$
I_{n}^{P}\left(T_{2}\right)=-\frac{\pi^{2} \sigma_{1}(n)}{2|n|}\left[\left(1-\frac{2}{5} \pi^{2} n^{2} T_{2}^{2}\right) \frac{\Psi(n)}{\left(2 \pi|n| T_{2}\right)^{3}}+4 \ln \left(2 \pi|n| T_{2}\right)\right]+O\left(T_{2} \ln T_{2}\right)
$$

where we have used (B.3), and kept all terms that diverge as $T_{2} \rightarrow 0$. Here $\Psi(n)$ is given by the expression

$$
\Psi(n)=-215-\frac{220}{|n|}+60 \ln (2 \pi|n|)+60(\gamma+\ln 2)\left(1+\frac{1}{|n|}\right) .
$$

Note that there is no $T_{2}^{-2}$ term in (2.21). Thus the cancellation of the $T_{2}^{-3}$ in the small $T_{2}$ expansion gives us that

$$
b_{n}=\frac{\pi^{2} \sigma_{1}(n) \Psi_{3, n}}{15|n|^{1 / 2}}
$$


yielding the complete solution. In fact the $T_{2}^{-1}$ term also cancels on adding (2.20) and (2.21), and hence the only singular term in $I_{n}\left(T_{2}\right)$ for small $T_{2}$ is given by

$$
-2 \pi^{2} \frac{\sigma_{1}(n)}{|n|} \ln \left(2 \pi|n| T_{2}\right) .
$$

Now for large $T_{2}, I_{n}\left(T_{2}\right)$ behaves as $e^{-2 \pi|n| T_{2}}$ with the leading contribution being given by

$$
I_{n}\left(T_{2}\right)=2 \pi^{2} \frac{\sigma_{1}(n)}{n^{2}} \ln \left(2 \pi|n| T_{2}\right) e^{-2 \pi|n| T_{2}}
$$

where we have used (B.2). Thus these are contributions from bound states of worldsheet instantons (or anti-instantons) if $n$ is positive (or negative).

\subsubsection{The solution for $I_{n_{1}, n_{2}}\left(T_{2}\right)$}

Like before, we express

$$
I_{n_{1}, n_{2}}\left(T_{2}\right)=I_{n_{1}, n_{2}}^{H}\left(T_{2}\right)+I_{n_{1}, n_{2}}^{P}\left(T_{2}\right),
$$

where $I_{n_{1}, n_{2}}^{H}\left(T_{2}\right)$ is a solution to the homogeneous equation (2.15), while $I_{n_{1}, n_{2}}^{P}\left(T_{2}\right)$ solves the particular equation (2.15).

The solution $I_{n_{1}, n_{2}}^{H}\left(T_{2}\right)$ satisfying the large $T_{2}$ boundary condition is given by

$$
I_{n_{1}, n_{2}}^{H}\left(T_{2}\right)=c_{n_{1}, n_{2}} \sqrt{T_{2}} K_{7 / 2}\left(2 \pi\left|n_{1}+n_{2}\right| T_{2}\right)
$$

where $c_{n_{1}, n_{2}}$ is an arbitrary constant.

We now consider the particular solution $I_{n_{1}, n_{2}}^{P}\left(T_{2}\right)$. It is convenient to consider the two cases separately:

(i) $n_{1}$ and $n_{2}$ have same sign (thus $n_{1} n_{2}>0$ ), and

(ii) $n_{1}$ and $n_{2}$ have opposite signs (thus $n_{1} n_{2}<0$ ).

For case (i), we have that

$$
I_{n_{1}, n_{2}}^{P}\left(T_{2}\right)=-\frac{6 \pi^{2} \sigma_{1}\left(n_{1}\right) \sigma_{1}\left(n_{2}\right)}{n_{1} n_{2} x^{3}} e^{-x}\left(2 x^{2}+5 x+5\right),
$$

where $x=2 \pi\left|n_{1}+n_{2}\right| T_{2}$. Unlike the other cases, there are no contributions involving the exponential integral function.

To determine $c_{n_{1}, n_{2}}$, we demand the cancellation of the $T_{2}^{-3}$ term in the small $T_{2}$ expansion of $I_{n_{1}, n_{2}}\left(T_{2}\right)$ as discussed earlier, which gives us that

$$
c_{n_{1}, n_{2}}=4 \pi^{2} \frac{\left|n_{1}+n_{2}\right|^{1 / 2}}{n_{1} n_{2}} \sigma_{1}\left(n_{1}\right) \sigma_{1}\left(n_{2}\right) .
$$

This also cancels the $T_{2}^{-1}$ term in the small $T_{2}$ expansion and hence there are no singular terms in $I_{n_{1}, n_{2}}\left(T_{2}\right)$ in this limit, as there is no $T_{2}^{-2}$ term that arises from (2.28).

On expanding $I_{n_{1}, n_{2}}\left(T_{2}\right)$ for large $T_{2}$, we see that all the terms are suppressed by a factor of $e^{-2 \pi\left|n_{1}+n_{2}\right| T_{2}}$. Hence they arise from bound states of worldsheet instantons 
or anti-instantons depending on whether $n_{1}$ is positive or negative. In fact, the leading contribution is given by

$$
\frac{2 \pi^{2} \sigma_{1}\left(n_{1}\right) \sigma_{1}\left(n_{2}\right)}{n_{1} n_{2}} e^{-2 \pi\left|n_{1}+n_{2}\right| T_{2}} .
$$

Now for case (ii), we have the particular solution

$$
\begin{aligned}
I_{n_{1}, n_{2}}^{P}\left(T_{2}\right)= & \frac{3 e^{-2 \pi\left(\left|n_{1}\right|+\left|n_{2}\right|\right) T_{2}} \sigma_{1}\left(n_{1}\right) \sigma_{1}\left(n_{2}\right)}{32 \pi^{2} n_{1} n_{2}\left|n_{1}+n_{2}\right|^{7} T_{2}^{3}}\left[( \alpha - \beta ) \left(5\left(\left|n_{1}\right|+\left|n_{2}\right|\right) R_{13,15}\right.\right. \\
& \left.+10 \pi\left(n_{1}+n_{2}\right)^{2} R_{3,5} T_{2}+4 \pi^{2}\left(n_{1}+n_{2}\right)^{2}\left(\left|n_{1}\right|+\left|n_{2}\right|\right) R_{1,5} T_{2}^{2}\right) \\
& -\frac{\alpha \beta}{2 \pi} R_{1,5}\left(P\left(2 \pi\left|n_{1}+n_{2}\right| T_{2}\right) e^{\alpha T_{2}} \operatorname{Ei}\left(-\alpha T_{2}\right)\right. \\
& \left.\left.-P\left(-2 \pi\left|n_{1}+n_{2}\right| T_{2}\right) e^{\beta T_{2}} \operatorname{Ei}\left(-\beta T_{2}\right)\right)\right],
\end{aligned}
$$

where $\alpha(>0)$ and $\beta$ are defined by

$$
\begin{aligned}
& \alpha=2 \pi\left(\left|n_{1}\right|+\left|n_{2}\right|-\left|n_{1}+n_{2}\right|\right), \\
& \beta=2 \pi\left(\left|n_{1}\right|+\left|n_{2}\right|+\left|n_{1}+n_{2}\right|\right),
\end{aligned}
$$

while $R_{a, b}$ is defined by

$$
R_{a, b}=a\left(n_{1}+n_{2}\right)^{2}-b\left(n_{1}-n_{2}\right)^{2} .
$$

Also $P(x)$ is the polynomial defined by (2.19).

In order to determine $c_{n_{1}, n_{2}}$, we cancel the $T_{2}^{-3}$ term in the small $T_{2}$ expansion of $I_{n_{1}, n_{2}}\left(T_{2}\right)$ as before. On using (B.3), the small $T_{2}$ expansion of (2.31) is given by

$$
I_{n_{1}, n_{2}}^{P}\left(T_{2}\right)=\frac{3 \sigma_{1}\left(n_{1}\right) \sigma_{1}\left(n_{2}\right)}{64 \pi^{3} n_{1} n_{2}\left|n_{1}+n_{2}\right|^{7} T_{2}^{3}}\left[1-\frac{2}{5} \pi^{2}\left(n_{1}+n_{2}\right)^{2} T_{2}^{2}\right] \Psi\left(n_{1}, n_{2}\right)+O\left(T_{2}^{0}\right)
$$

where

$$
\Psi\left(n_{1}, n_{2}\right)=-15 \alpha \beta R_{1,5} \ln (\alpha / \beta)+\frac{5}{2}\left(\alpha^{2}-\beta^{2}\right) R_{13,15} .
$$

We note that the $T_{2}^{-2}$ term vanishes in (2.34). Thus we have that

$$
c_{n_{1}, n_{2}}=-\frac{\sigma_{1}\left(n_{1}\right) \sigma_{1}\left(n_{2}\right) \Psi\left(n_{1}, n_{2}\right)}{20 n_{1} n_{2}\left|n_{1}+n_{2}\right|^{7 / 2}} .
$$

In fact, the $T_{2}^{-1}$ term also cancels in the small $T_{2}$ expansion of $I_{n_{1}, n_{2}}\left(T_{2}\right)$ and hence there are no singular terms in this expansion.

Now consider the large $T_{2}$ expansion of $I_{n_{1}, n_{2}}\left(T_{2}\right)$. For fixed $n_{1}$ and $n_{2}$, the leading contribution comes from the homogeneous solution and is of the form $e^{-2 \pi\left|n_{1}+n_{2}\right| T_{2}}$. Thus the leading contribution is given by

$$
-\frac{\sigma_{1}\left(n_{1}\right) \sigma_{1}\left(n_{2}\right)}{40 n_{1} n_{2}\left(n_{1}+n_{2}\right)^{4}} \Psi\left(n_{1}, n_{2}\right) e^{-2 \pi\left|n_{1}+n_{2}\right| T_{2}} .
$$


The particular solution is exponentially suppressed by an additional factor of $e^{-\alpha T_{2}}$, and the leading contribution is given by

$$
-\frac{3 \sigma_{1}\left(n_{1}\right) \sigma_{1}\left(n_{2}\right)}{2 n_{1}^{2} n_{2}^{2} T_{2}^{2}} e^{-2 \pi\left(\left|n_{1}\right|+\left|n_{2}\right|\right) T_{2}}
$$

on using (B.2).

Contributions of this kind that are exponentially suppressed at large $T_{2}$ arise from bound states of worldsheet instantons and anti-instantons.

Thus the above expressions yield the complete data needed to evaluate (2.13). Now in (2.13), the contributions arising from $n_{1} n_{2}<0$ yield an infinite sum given by

$$
2 \sum_{n_{1} \geq n+1} I_{n_{1}, n-n_{1}}
$$

and hence it is worthwhile to check the convergence of this sum. For this, we focus on the large $n_{1}$ behavior of the various terms while keeping $n$ fixed. The contribution arising from the particular solution (2.31) is exponentially damped in this limit, hence convergence is trivial. To analyze the contributions that arise from the homogeneous solution (2.27) consider the large $n_{1}$ limit of $c_{n_{1}, n-n_{1}}$ in (2.36). This is given by

$$
c_{n_{1}, n-n_{1}} \rightarrow \frac{4 \pi^{2} \sigma_{1}\left(n_{1}\right)^{2}|n|^{7 / 2}}{35\left|n_{1}\right|^{5}}
$$

as several leading contributions cancel. Using the inequality [25]

$$
\sigma_{1}(n)<e^{\gamma} n \ln \ln n+\frac{0.6483 n}{\ln \ln n}
$$

for $n \geq 3$, it follows that the sum over $n_{1}$ is convergent. $^{9}$

\section{S-duality and an elementary consequence for $D$ string instanton contributions}

The worldsheet instanton contributions under S-duality get mapped to $D$ string instanton contributions [1, 27]. Given the exact expressions for the worldsheet instanton contributions, though it takes work to implement S-duality in order to obtain the complete $D$ string instanton contributions, it is elementary to implement strong weak coupling duality to obtain a part of the $D$ string instanton contributions, which we now illustrate.

\footnotetext{
${ }^{9} \mathrm{~A}$ related inequality is given by $[26]$

$$
\sigma_{1}(n)<H_{n}+e^{H_{n}} \ln H_{n},
$$
}

for $n>1$, where $H_{n}$ is the $n$th harmonic number. Using the asymptotic expansion for $H_{n}$ given by

$$
H_{n}=\ln n+\gamma+\frac{1}{2 n}-\sum_{m=1}^{\infty} \frac{B_{2 m}}{2 m \cdot n^{2 m}}
$$

where $B_{m}$ are the Bernoulli numbers, we again see that the sum over $n_{1}$ in $(2.40)$ is convergent. 
As a simple example, consider the worldsheet instanton contribution to the $\mathcal{R}^{4}$ coupling given by

$$
2 \pi \sum_{n=1}^{\infty} \frac{\sigma_{1}(n)}{n} e^{2 \pi i n T}
$$

which follows from (1.6). In the background where $\tau_{1}=0$, strong weak coupling duality yields

$$
\tau_{2} \rightarrow \frac{1}{\tau_{2}}, \quad V \rightarrow \tau_{2} V, \quad B_{N} \rightarrow B_{R}, \quad B_{R} \rightarrow-B_{N} .
$$

Thus performing the S-duality transformation (3.2) on (3.1), we get the $D$ string instanton contribution $^{10}$

$$
2 \pi \sum_{n=1}^{\infty} \frac{\sigma_{1}(n)}{n} e^{2 \pi i n S}
$$

where

$$
S=S_{1}+i S_{2}=B_{R}+i \tau_{2} V
$$

Similarly for the $D^{6} \mathcal{R}^{4}$ coupling the S-duality transformations (3.2) yield partial contributions to the $D$ string instanton contributions using the various expressions for the worldsheet instanton contributions we have analyzed. For example, from (2.6) we see that the contribution from the bound states of $D$ string instantons/anti-instantons carrying no net $\mathrm{RR}$ charge is given by

$$
\sum_{n=1}^{\infty} \widetilde{Q}_{n}\left(S_{2}\right) e^{-4 \pi n S_{2}}
$$

where $\widetilde{Q}_{n}\left(S_{2}\right)$ is given by

$$
\widetilde{Q}_{n}\left(S_{2}\right)=-\frac{3 \sigma_{1}(n)^{2}}{n^{4} S_{2}^{2}}\left[1-\frac{4}{y}+\frac{1}{168} \sum_{m=0}^{\infty} \frac{(-1)^{m}(m+7) !}{y^{m+2}}\right],
$$

where $y=4 \pi n S_{2}$ and we have performed a weak coupling (large $\tau_{2}$ ) expansion using (B.2). While the overall $S_{2}$ dependence must arise from the structure of zero modes in the instanton/anti-instanton background, we see that the infinite sum is an expansion in $y \sim e^{-\phi}$, the open string coupling. Note that performing (3.2) on $I_{n}^{P}\left(T_{2}\right)$ in (2.18) yields contributions having factors of $\ln \tau_{2}$, which arise from non-local interactions logarithmic in the external momenta in the string frame, on converting to the Einstein frame. This is precisely what is expected from the structure of the U-duality invariant eigenvalue equation that arises for the $D^{6} \mathcal{R}^{4}$ coupling $[4,6]$, as the source term contains $\ln \tau_{2}$ that arises from the $\mathcal{R}^{4}$ coupling [2].

\footnotetext{
${ }^{10}$ In fact this is the complete answer from the sum over the $(0, n) D$ string instantons which follows from the U-duality invariant expression for the $\mathcal{R}^{4}$ coupling [2, 3].
} 


\section{A The $\mathrm{SL}(2, \mathbb{Z})$ invariant non-holomorphic Eisenstein series}

The non-holomorphic Eisenstein series $E_{s}(T, \bar{T})$ is given by the expression

$$
\begin{aligned}
E_{s}(T, \bar{T})= & 2 \zeta(2 s) T_{2}^{s}+2 \sqrt{\pi} T_{2}^{1-s} \frac{\Gamma(s-1 / 2)}{\Gamma(s)} \zeta(2 s-1) \\
& +\frac{4 \pi^{s} \sqrt{T_{2}}}{\Gamma(s)} \sum_{n \neq 0} \frac{\sigma_{2 s-1}(n)}{|n|^{s-1 / 2}} K_{s-1 / 2}\left(2 \pi|n| T_{2}\right) e^{2 \pi i n T_{1}}
\end{aligned}
$$

on expanding around large $T_{2}$. Here the divisor function $\sigma_{m}(n)$ is defined by

$$
\sigma_{m}(n)=\sum_{d \mid n, d>0} d^{m},
$$

where the sum is over the positive divisors of $n$. The case $s=1$ has to be regularized and is given by

$$
\begin{aligned}
E_{1}(T, \bar{T}) & =-\pi \ln \left(T_{2}|\eta(T)|^{4}\right) \\
& =\frac{\pi^{2}}{3} T_{2}-\pi \ln T_{2}+2 \pi \sum_{n \neq 0} \frac{\sigma_{1}(n)}{|n|} e^{2 \pi i\left(n T_{1}+i|n| T_{2}\right)}
\end{aligned}
$$

\section{B The exponential integral function}

The exponential integral function $\operatorname{Ei}(-x)$ is given by the integral representation

$$
e^{x} \operatorname{Ei}(-x)=-\frac{1}{x}+\int_{0}^{\infty} d t \frac{e^{-t}}{(t+x)^{2}}, \quad x>0 .
$$

Thus we see that $e^{x} \operatorname{Ei}(-x)$ is a polynomial in $1 / x$ of the form

$$
e^{x} \operatorname{Ei}(-x)=-\frac{1}{x}+\sum_{n=0}^{\infty} \frac{(-1)^{n}(n+1) !}{x^{n+2}}
$$

for large $x$. On the other hand, for small $x$, the series expansion is given by

$$
\operatorname{Ei}(-x)=\gamma+\ln x+\sum_{n=1}^{\infty} \frac{(-x)^{n}}{n \cdot n !}, \quad x>0 .
$$

Open Access. This article is distributed under the terms of the Creative Commons Attribution License (CC-BY 4.0), which permits any use, distribution and reproduction in any medium, provided the original author(s) and source are credited.

\section{References}

[1] J.H. Schwarz, An $\mathrm{SL}(2, \mathbb{Z})$ multiplet of type IIB superstrings, Phys. Lett. B 360 (1995) 13 [Erratum ibid. B 364 (1995) 252] [hep-th/9508143] [INSPIRE].

[2] E. Kiritsis and B. Pioline, On $R^{4}$ threshold corrections in IIB string theory and $(p, q)$ string instantons, Nucl. Phys. B 508 (1997) 509 [hep-th/9707018] [INSPIRE]. 
[3] M.B. Green, M. Gutperle and P. Vanhove, One loop in eleven-dimensions, Phys. Lett. B 409 (1997) 177 [hep-th/9706175] [INSPIRE].

[4] M.B. Green, J.G. Russo and P. Vanhove, Automorphic properties of low energy string amplitudes in various dimensions, Phys. Rev. D 81 (2010) 086008 [arXiv:1001.2535] [INSPIRE].

[5] A. Basu, The $D^{4} R^{4}$ term in type IIB string theory on $T^{2}$ and $U$-duality, Phys. Rev. D 77 (2008) 106003 [arXiv:0708.2950] [INSPIRE].

[6] A. Basu, The $D^{6} R^{4}$ term in type IIB string theory on $T^{2}$ and $U$-duality, Phys. Rev. $\mathbf{D} 77$ (2008) 106004 [arXiv:0712.1252] [InSPIRE].

[7] M.B. Green and P. Vanhove, The Low-energy expansion of the one loop type-II superstring amplitude, Phys. Rev. D 61 (2000) 104011 [hep-th/9910056] [INSPIRE].

[8] H. Gomez and C.R. Mafra, The closed-string 3-loop amplitude and S-duality, JHEP 10 (2013) 217 [arXiv: 1308.6567] [INSPIRE].

[9] E. D'Hoker, M.B. Green, B. Pioline and R. Russo, Matching the $D^{6} R^{4}$ interaction at two-loops, JHEP 01 (2015) 031 [arXiv:1405.6226] [INSPIRE].

[10] A. Basu, Perturbative type-II amplitudes for BPS interactions, Class. Quant. Grav. 33 (2016) 045002 [arXiv: 1510.01667] [INSPIRE].

[11] B. Pioline and R. Russo, Infrared divergences and harmonic anomalies in the two-loop superstring effective action, JHEP 12 (2015) 102 [arXiv:1510.02409] [INSPIRE].

[12] M.B. Green and P. Vanhove, Duality and higher derivative terms in M-theory, JHEP 01 (2006) 093 [hep-th/0510027] [INSPIRE].

[13] A. Basu and S. Sethi, Recursion Relations from Space-time Supersymmetry, JHEP 09 (2008) 081 [arXiv:0808.1250] [INSPIRE].

[14] G. Bossard and V. Verschinin, Minimal unitary representations from supersymmetry, JHEP 10 (2014) 008 [arXiv: 1406.5527] [INSPIRE].

[15] A. Basu, The $D^{6} R^{4}$ term from three loop maximal supergravity, Class. Quant. Grav. 31 (2014) 245002 [arXiv: 1407.0535] [INSPIRE].

[16] B. Pioline, $D^{6} R^{4}$ amplitudes in various dimensions, JHEP 04 (2015) 057 [arXiv: 1502.03377] [INSPIRE].

[17] Y. Wang and X. Yin, Constraining Higher Derivative Supergravity with Scattering Amplitudes, Phys. Rev. D 92 (2015) 041701 [arXiv:1502.03810] [INSPIRE].

[18] G. Bossard and V. Verschinin, The two $\nabla^{6} R^{4}$ type invariants and their higher order generalisation, JHEP 07 (2015) 154 [arXiv: 1503.04230] [INSPIRE].

[19] G. Bossard and A. Kleinschmidt, Loops in exceptional field theory, JHEP 01 (2016) 164 [arXiv: 1510.07859] [INSPIRE].

[20] G. Bossard and A. Kleinschmidt, Cancellation of divergences up to three loops in exceptional field theory, JHEP 03 (2018) 100 [arXiv: 1712.02793] [INSPIRE].

[21] O. Ahlén and A. Kleinschmidt, $D^{6} R^{4}$ curvature corrections, modular graph functions and Poincaré series, JHEP 05 (2018) 194 [arXiv: 1803.10250] [INSPIRE].

[22] D. Dorigoni and A. Kleinschmidt, Modular graph functions and asymptotic expansions of Poincaré series, Commun. Num. Theor. Phys. 13 (2019) 569 [arXiv:1903.09250] [InSPIRE]. 
[23] G. Bossard, A. Kleinschmidt and B. Pioline, 1/8-BPS Couplings and Exceptional Automorphic Functions, arXiv:2001.05562 [INSPIRE].

[24] M.B. Green, S.D. Miller and P. Vanhove, $\mathrm{SL}(2, \mathbb{Z})$-invariance and D-instanton contributions to the $D^{6} R^{4}$ interaction, Commun. Num. Theor. Phys. 09 (2015) 307 [arXiv:1404.2192] [INSPIRE].

[25] G. Robin, Large values of the sum-of-divisors function and the Riemann hypothesis, J. Math. Pures Appl. 63 (1984) 187.

[26] J.C. Lagarias, An Elementary Problem Equivalent to the Riemann Hypothesis, Am. Math. Mon. 109 (2002) 534 [math/0008177].

[27] E. Witten, Bound states of strings and p-branes, Nucl. Phys. B 460 (1996) 335 [hep-th/9510135] [INSPIRE]. 